\title{
AN ENQUIRY ON ICHTHYODIVERSITY OF THE CHALAN BEEL, A KEY ICHTHYOFAUNAL HOTSPOT OF NORTHWESTERN BANGLADESH
}

\author{
Gawsia Wahidunnessa Chowdhury", Nime Sarker, Muntasir Akash and Gulshan Ara Latifa \\ Department of Zoology, University of Dhaka, Dhaka, Bangladesh \\ *Email: gawsia@gmail.com, gawsia@du.ac.bd
}

\begin{abstract}
This meta-analysis focused on the drift in ichthyodiversity of the Chalan Beel, a marshy, natural inland indentation, the largest of its kind in northwest (NW) Bangladesh. To apprehend the objective, we considered peer-reviewed fish inventories of the area published since 1971. The first checklist on the fishes of the Chalan Beel was compiled in 2009 after which, till now, we found only four similar studies. In total, we found 139 species annotated in these works including 10 exotic species. We, however, surmised a downward trend in fish fauna; from 114 species listed in 2009, in 2017 the Chalan Beel is reported to have 66 species - comprising only 30 species common in each of the five works. Cyprinidae is turned out as the most diverse abundant family for the area with 43 species. The most diverse order, on the contrary, is Siluriformes represented by 10 families and 42 species. Of the fish inventory, 3 were Critically Endangered, 15 Endangered, 13 Vulnerable and 21 Near Threatened in Bangladesh. The Chalan Beel is reported to have 3 globally Vulnerable and 10 globally Near Threatened fish. Reviewing works on the Chalan Beel revealed a potential new exotic species Trichogaster labiosa for Bangladesh and mentioning of 3 species found in no national fish inventories viz., Erethistoides infuscatus, Parachiloglanis hodgarti and Poropuntius clavatus.
\end{abstract}

Key words: Chalan beel, Ichthyodiversity, Bangladesh, Hotspot, Ganges, Brahmaputra.

\section{INTRODUCTION}

Chipped between two of the most extensive and intricate drainage systems of the Earth - the Brahmaputra and the Ganges, the lower northwestern Bangladesh is characterized by a cluster of permanent, naturally depressed smaller catchment areas, fed by tributary run-offs and downpour, which turns into a vast, singular waterbody in monsoon. The whole network of inundations is collectively known as the Chalan
Beel, comprising a low-lying floodplain area of about $375 \mathrm{~km}^{2}$ and about $52-78 \mathrm{~km}^{2}$ area of persistent waterbodies. Pristine zone of this system is considered shrunk to only $26 \mathrm{~km}^{2}$ (Alam and Hossain 2003, Hossain et al. 2009). The Chalan Beel touchdowns three districts of Natore, Pabna and Sirajganj and 10 subdistricts between $24.35^{\circ}$ to $24.70^{\circ} \mathrm{N}$ Latitude and between $89.10^{\circ}$ to $89.35^{\circ} \mathrm{E}$ Longitude, in past, it is claimed to be spanned across six districts covering more than $1000 \mathrm{~km}^{2}$ (Fig. 1). 
The Chalan beel empties into the Ganges and Brahmaputra Rivers through the Baral River on the south (Hossain et al. 2009). This depression is formed by the merging of dried up former courses of the Atrai River and the Karatoya River - two major tributaries of the Brahmaputra River in northern Bangladesh (Hossain et al. 2009).

Several decades ago, this beel was rich with variety of fishes. But in recent years, aquatic resources of this water body are decreasing because of uncontrolled fishing and environmental resources (Alam and Hossain 2003, Galib et al. 2009).

As per of the most recent inventories, 251-260 species of indigenous freshwater fishes are documented by means of voucher specimens together with/or by photographic records (Siddqui et al. 2008, IUCN Bangladesh 2015, DoF 2016). Freshwater fish species in Bangladesh are constantly on the increase owing to the prowess of advanced taxonomic study and sampling effort ( $\mathrm{Ng}$ 2005, Kullander et al. 2015, Rahman et al. 2016). The actual species richness is considered to be much higher being the whole country is placed on the largest deltaic plain of the planet.

Regarding the fish diversity of Bangladesh faced with regular discoveries, the Chalan Beel stands as a least explored region. This retrospective attempted a track down of fish inventories of the Chalan Beel so far compiled in order to visualizing the present situation and drift in ichthyodiversity in recent years. We believe that, in turn, this work will help devising conservation and management outlines for fisheries resources of the Chalan Beel.

\section{MATERIALS AND METHODS}

Only peer-reviewed publications on the fish diversity of the Chalan Beel were considered for this meta-analysis. Works were skimmed out by using the Google Scholar search tool. Keywords applied for the search were: ichthyofauna, ichthyodiversity, Chalan Beel and Bangladesh. This review has been restricted by time duration between 1971 and 2018 .

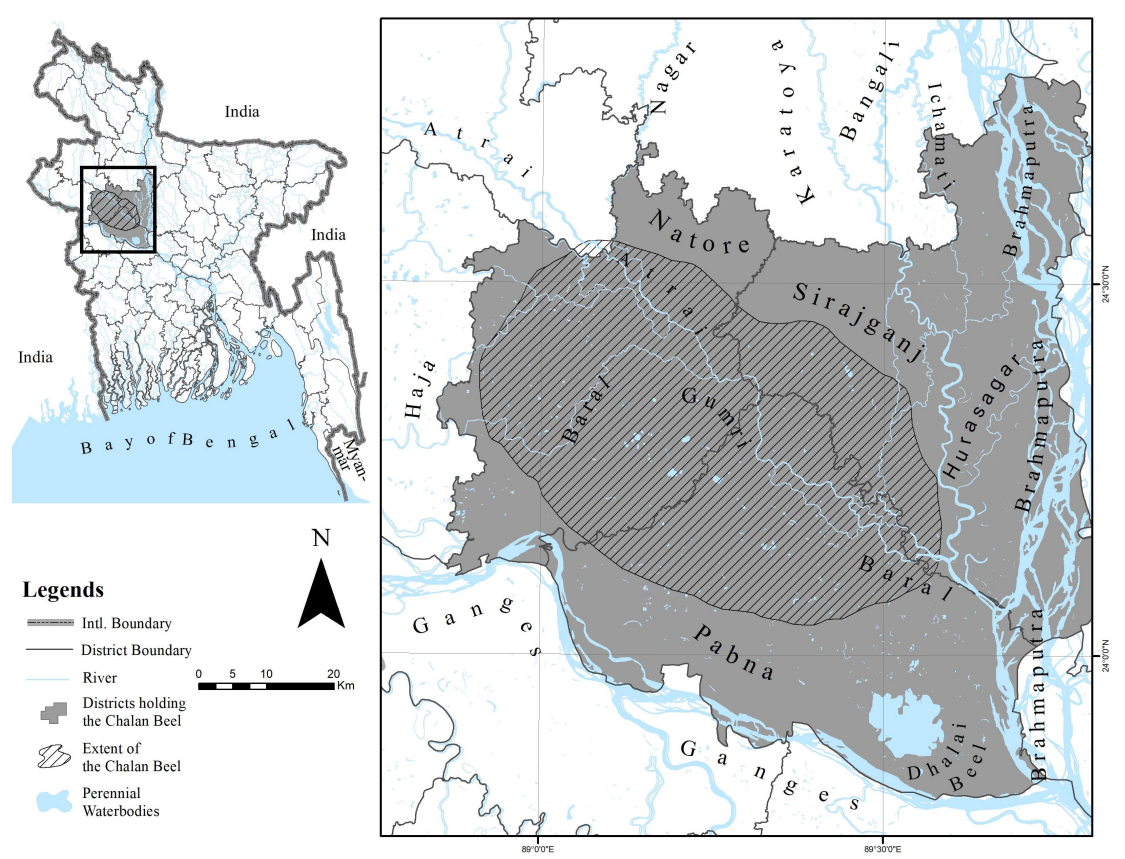

Figure 1. Extent of the Chalan beel in Bangladesh. 
We, from the whole search process, deemed five studies for the review Hossain et al. (2009), Galib et al. (2009), Kostori et al. (2011), Siddique et al. (2016), Rahman et al. (2017) (Table 1).

For using the most updated naming convention and avoiding synonymies, Nelson et al. (2016) and Eschmeyer and Fong (2018) were consulted. Synonyms or invalid taxa were ruled out or merged into the valid ones. For example, we did not consider Colisa sota noted by Kostori et al. (2011) as the taxon is no more valid and has now been considered as Trichogaster chuna.

\section{RESULTS AND DISCUSSIONS}

In total, five inventories on the Chalan Beel enlisted 139 different species from 35 families with a notation of 10 exotic species. Summary on taxonomic viewpoint is provided on Table 1.

From Table 1, it is evident that, in single study attempt, fish species richness of the Chalan Beel stood the peak in Hossain et al. (2009). Gradually, afterwards, the richness exhibited demise in diversity. Rahman et al. (2017) yielded the least diverse result yet being the most recent.

Considering the frequency of appearance of fish species in the works, we found 30 species in all five, 29 from four, 17 from three, 2 from 20 and 36 from only one study (Fig.2, Table 1).
From Table 1, the carp family Cyprinidae appeared to be the most abundant represented by 38 indigenous and 5 exotic species. Considering taxon above family, the catfish order Siluriformes is the largest for the Chalan Beel showing 10 distinct families and 42 species. However, catfishes are relatively rarer than the cyprinids in terms of frequency of appearance in inventories.

We also checked the global and national status of the fishes of the Chalan Beel. We found 3 Critically Endangered, 15 Endangered, 13 Vulnerable and 21 Near Threatened species, of which 3 were globally Vulnerable and 10 were globally Near Threatened fish (IUCN Bangladesh 2015), (Fig. 3). Of globally Vulnerable species, the Chalan Beel harbors stocks of Cirrhinus cirrhosus, Botia rostrata and Monopterus cuchia (Table 1).

Labeo boga and two catfish species Ompok pabo and Bagarius bagarius are the three nationally Critically Endangered species of the Chalan Beel. The inventories also mentioned 10 globally and 21 nationally Near Threatened species as well as 5 globally and 6 nationally Data Deficient species from time to time. Though majority of species are Least Concern here, the Chalan Beel is also found to be a good harbor of cryptic and least known species being documented with 16 globally and 8 nationally Not Evaluated species (Froese and Pauli 2018) (Fig. 3).

Table 1. Summary of the fish inventories of the Chalan Beel, Bangladesh

\begin{tabular}{lccc}
\hline Works & Species & Family & Order \\
\hline Rahman et al. $(2017)$ & 66 & 23 & 8 \\
Siddique et al. $(2016)$ & 78 & 26 & 10 \\
Kostori et al. $(2011)$ & 82 & 22 & 10 \\
Galib et al. $(2009)$ & 81 & 27 & 12 \\
Hossain et al. $(2009)$ & 114 & 29 & 13 \\
\hline
\end{tabular}




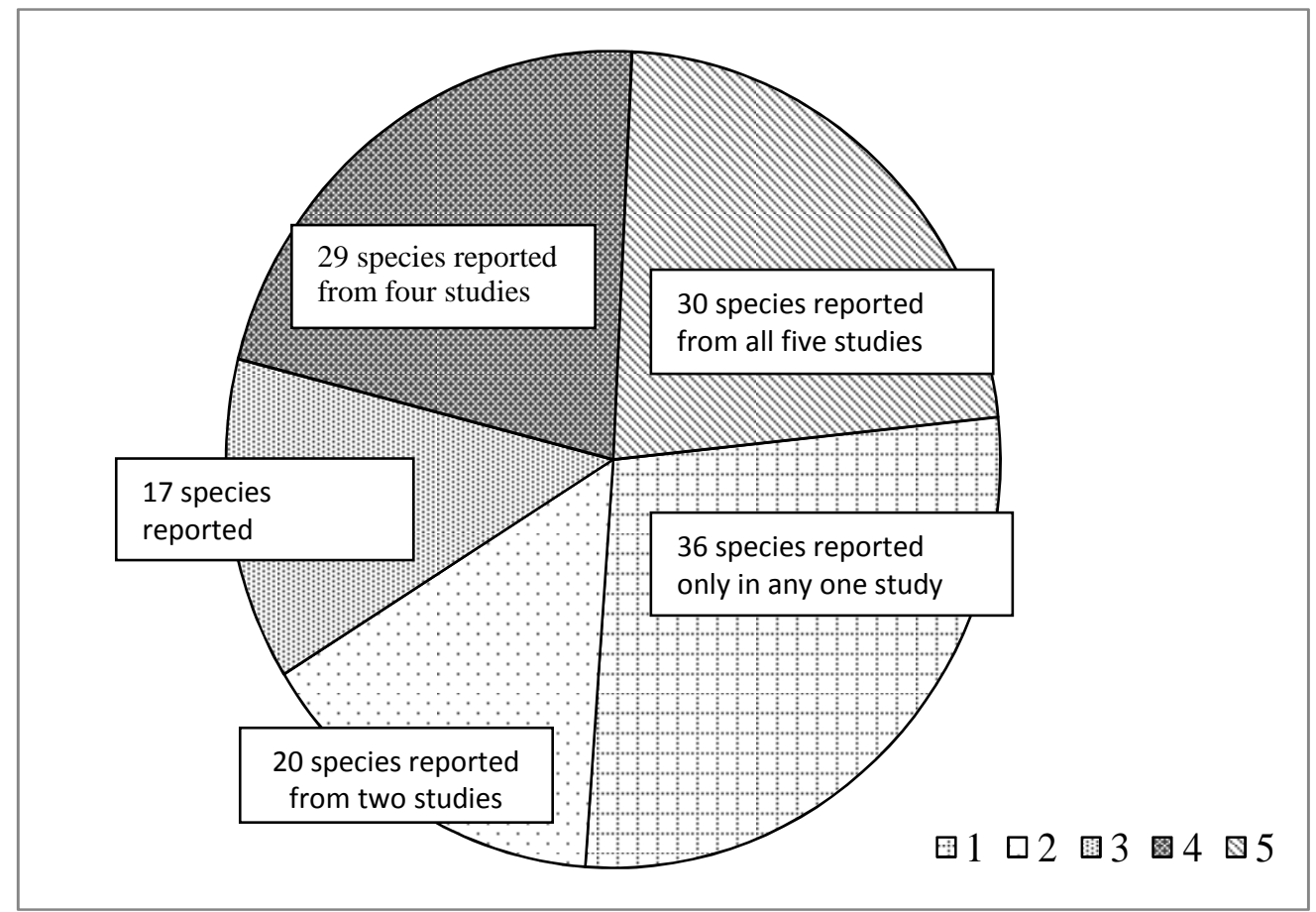

Figure 2. A comparison of commonness among the five inventories.

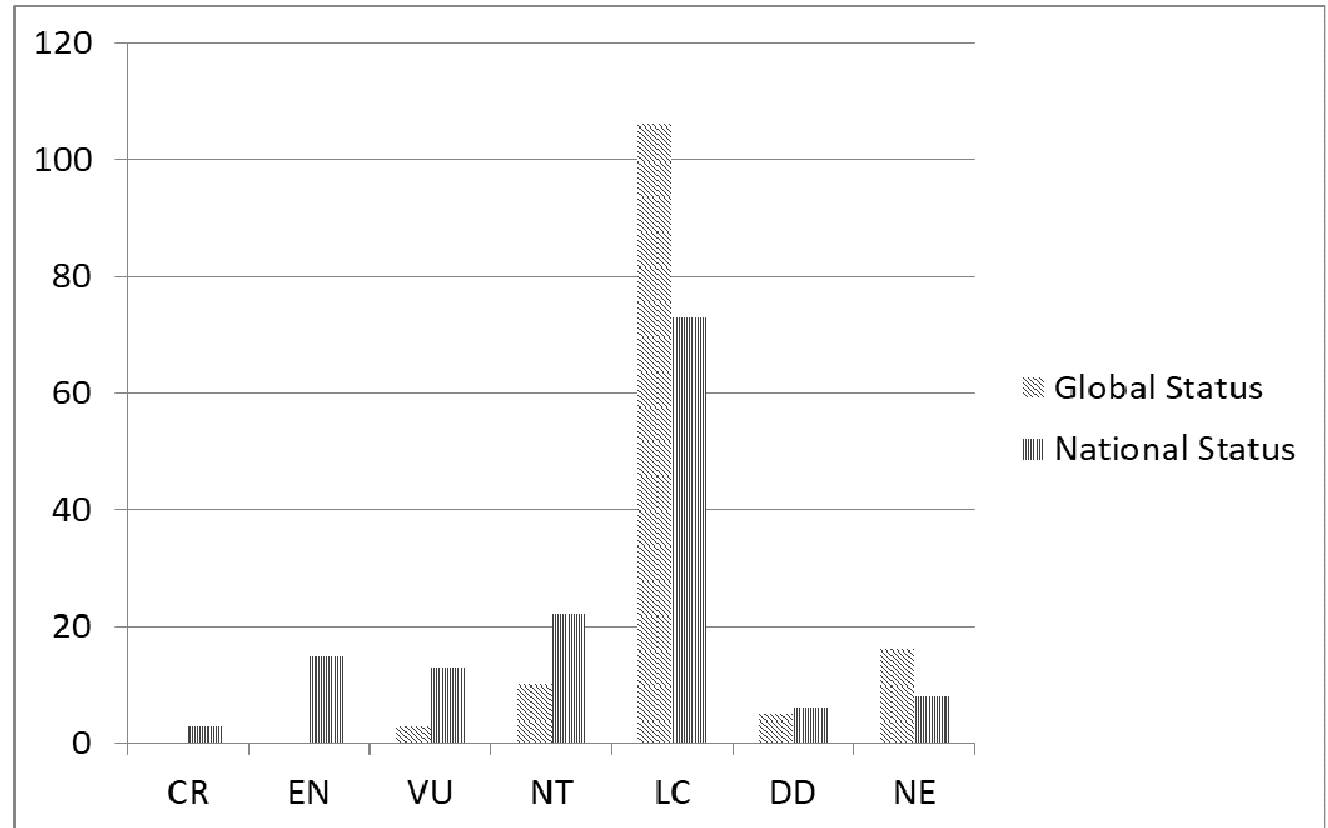

Figure 3. Conservation status of fish species reported from the Chalan Beel.

Note: $\mathrm{CR}=$ Critically Endangered, $\mathrm{EN}=$ Endangered, $\mathrm{VU}=$ Vulnerable, $\mathrm{NT}=$ Near Threatened, $\mathrm{LC}=$ Least Concern, $\mathrm{DD}=$ Data Deficient, $\mathrm{VU}=$ Vulnerable, $\mathrm{NE}=$ Not Evaluated 
Table 2. List of indigenous fish species recorded from Chalan Beel, Bangladesh.

CR - Critically Endangered; EN - Endangered; VU - Vulnerable; NT - Near Threatened;

LC - Least Concern; DD - Data Deficient; NE - Not Evaluated

\begin{tabular}{|c|c|c|c|c|c|c|c|c|}
\hline Family & Species & $\begin{array}{l}\text { National } \\
\text { Status }\end{array}$ & $\begin{array}{l}\text { Global } \\
\text { Status }\end{array}$ & $\begin{array}{l}\text { Galib } \\
\text { et al. } \\
2009 \\
\end{array}$ & $\begin{array}{c}\text { Hossain } \\
\text { et al. } \\
2009 \\
\end{array}$ & $\begin{array}{c}\text { Kostori } \\
\text { et al. } \\
2011 \\
\end{array}$ & $\begin{array}{c}\text { Siddiqui } \\
\text { et al. } \\
2016 \\
\end{array}$ & $\begin{array}{c}\text { Rahman } \\
\text { et al. } \\
2017 \\
\end{array}$ \\
\hline Anguillidae & $\begin{array}{l}\text { Anguilla bengalensis } \\
\text { bengalensis }\end{array}$ & VU & NT & & $\checkmark$ & & & \\
\hline Muraenesocidae & Congresox talabon & $\mathrm{NE}$ & LC & & $\checkmark$ & & & \\
\hline \multirow[t]{2}{*}{ Ophichthidae } & $\begin{array}{l}\text { Pisodonophis } \\
\text { cancrivorus }\end{array}$ & $\mathrm{NE}$ & $\mathrm{LC}$ & $\checkmark$ & & & & \\
\hline & Pisodonophis boro & $\mathrm{LC}$ & $\mathrm{LC}$ & & $\checkmark$ & & & \\
\hline Synbranchidae & Monopterus cuchia & VU & VU & $\checkmark$ & $\checkmark$ & & $\checkmark$ & $\checkmark$ \\
\hline \multirow[t]{2}{*}{ Notopteridae } & Chitala chitala & EN & NT & $\checkmark$ & $\checkmark$ & & $\checkmark$ & $\checkmark$ \\
\hline & $\begin{array}{l}\text { Notopterus } \\
\text { notopterus }\end{array}$ & VU & $\mathrm{LC}$ & $\checkmark$ & $\checkmark$ & & $\checkmark$ & $\checkmark$ \\
\hline \multirow[t]{5}{*}{ Clupeidae } & Corica soborna & $\mathrm{LC}$ & $\mathrm{LC}$ & $\checkmark$ & & $\checkmark$ & & $\checkmark$ \\
\hline & Gonialosa manmina & $\mathrm{LC}$ & $\mathrm{LC}$ & & $\checkmark$ & & & \\
\hline & Gudusia chapra & VU & $\mathrm{LC}$ & $\checkmark$ & $\checkmark$ & $\checkmark$ & $\checkmark$ & $\checkmark$ \\
\hline & Gudusia variegata & $\mathrm{NE}$ & $\mathrm{LC}$ & & & $\checkmark$ & & \\
\hline & Tenualosa ilisha & $\mathrm{LC}$ & $\mathrm{LC}$ & $\checkmark$ & $\checkmark$ & & & \\
\hline \multirow[t]{2}{*}{ Engraulidae } & Setipinna phasa & $\mathrm{LC}$ & $\mathrm{LC}$ & & $\checkmark$ & $\checkmark$ & & \\
\hline & Setipinna taty & $\mathrm{LC}$ & $\mathrm{NE}$ & & $\checkmark$ & $\checkmark$ & & \\
\hline \multirow[t]{3}{*}{ Botiidae } & Botia dario & EN & $\mathrm{LC}$ & $\checkmark$ & $\checkmark$ & $\checkmark$ & $\checkmark$ & $\checkmark$ \\
\hline & Botia dayi & EN & $\mathrm{NE}$ & & $\checkmark$ & $\checkmark$ & & \\
\hline & Botia lohachata & EN & $\mathrm{NE}$ & $\checkmark$ & $\checkmark$ & $\checkmark$ & $\checkmark$ & \\
\hline Botiidae & Botia rostrata & DD & VU & & $\checkmark$ & & & \\
\hline \multirow[t]{4}{*}{ Cobitidae } & $\begin{array}{l}\text { Lepidocephalichthys } \\
\text { berdmorei }\end{array}$ & $\mathrm{LC}$ & $\mathrm{LC}$ & & & $\checkmark$ & $\checkmark$ & \\
\hline & $\begin{array}{l}\text { Lepidocephalichthys } \\
\text { guntea }\end{array}$ & $\mathrm{LC}$ & $\mathrm{LC}$ & $\checkmark$ & $\checkmark$ & $\checkmark$ & $\checkmark$ & $\checkmark$ \\
\hline & $\begin{array}{l}\text { Canthophrys } \\
\text { gongota }\end{array}$ & NT & $\mathrm{LC}$ & $\checkmark$ & $\checkmark$ & & $\checkmark$ & \\
\hline & Pangio pangia & $\mathrm{LC}$ & $\mathrm{LC}$ & & & $\checkmark$ & & \\
\hline \multirow[t]{2}{*}{ Nemacheildae } & Acanthocobitis botia & $\mathrm{LC}$ & $\mathrm{LC}$ & $\checkmark$ & $\checkmark$ & $\checkmark$ & $\checkmark$ & \\
\hline & Schistura savona & NT & $\mathrm{LC}$ & & & $\checkmark$ & & \\
\hline Aplocheilidae & Aplocheilus panchax & $\mathrm{LC}$ & $\mathrm{LC}$ & $\checkmark$ & $\checkmark$ & $\checkmark$ & $\checkmark$ & \\
\hline \multirow[t]{12}{*}{ Cyprinidae } & $\begin{array}{l}\text { Amblypharyngodon } \\
\text { microlepis }\end{array}$ & $\mathrm{LC}$ & $\mathrm{LC}$ & & & $\checkmark$ & & \\
\hline & $\begin{array}{l}\text { Amblypharyngodon } \\
\text { mola }\end{array}$ & $\mathrm{LC}$ & $\mathrm{LC}$ & & $\checkmark$ & $\checkmark$ & $\checkmark$ & $\checkmark$ \\
\hline & Aspidoparia jaya & $\mathrm{LC}$ & NE & & $\checkmark$ & $\checkmark$ & & \\
\hline & Aspidoparia morar & VU & $\mathrm{NE}$ & $\checkmark$ & $\checkmark$ & $\checkmark$ & & \\
\hline & Catla catla & $\mathrm{LC}$ & $\mathrm{NE}$ & $\checkmark$ & $\checkmark$ & & $\checkmark$ & $\checkmark$ \\
\hline & Cirrhinus cirrhosus & NT & VU & $\checkmark$ & $\checkmark$ & & $\checkmark$ & $\checkmark$ \\
\hline & Cirrhinus reba & NT & $\mathrm{LC}$ & $\checkmark$ & & $\checkmark$ & $\checkmark$ & $\checkmark$ \\
\hline & Chagunius chagunio & VU & $\mathrm{LC}$ & & $\checkmark$ & & & \\
\hline & Chela cachius & VU & $\mathrm{LC}$ & & $\checkmark$ & $\checkmark$ & & \\
\hline & Chela laubuca & $\mathrm{LC}$ & $\mathrm{NE}$ & & $\checkmark$ & $\checkmark$ & & \\
\hline & Danio rerio & NT & $\mathrm{LC}$ & & $\checkmark$ & $\checkmark$ & $\checkmark$ & \\
\hline & Devario devario & $\mathrm{LC}$ & $\mathrm{LC}$ & $\checkmark$ & & $\checkmark$ & $\checkmark$ & \\
\hline \multirow[t]{2}{*}{ Cyprinidae } & Esomus danricus & $\mathrm{LC}$ & $\mathrm{LC}$ & $\checkmark$ & $\checkmark$ & $\checkmark$ & $\checkmark$ & $\checkmark$ \\
\hline & Labeo ariza & $\mathrm{LC}$ & $\mathrm{LC}$ & & $\checkmark$ & & & \\
\hline
\end{tabular}

ECOPRINT VOL 24, 2017 


\begin{tabular}{|c|c|c|c|c|c|c|c|c|}
\hline & Labeo bata & $\mathrm{LC}$ & $\mathrm{LC}$ & $\checkmark$ & $\checkmark$ & $\checkmark$ & $\checkmark$ & \\
\hline & Labeo boga & $\mathrm{CR}$ & $\mathrm{LC}$ & & $\checkmark$ & $\checkmark$ & $\checkmark$ & \\
\hline & Labeo calbasu & $\mathrm{LC}$ & $\mathrm{LC}$ & $\checkmark$ & $\checkmark$ & & $\checkmark$ & $\checkmark$ \\
\hline & Labeo gonius & NT & $\mathrm{LC}$ & & $\checkmark$ & $\checkmark$ & & $\checkmark$ \\
\hline & Labeo rohita & $\mathrm{LC}$ & $\mathrm{LC}$ & $\checkmark$ & $\checkmark$ & & $\checkmark$ & $\checkmark$ \\
\hline & Megarasbora elanga & $\mathrm{EN}$ & $\mathrm{LC}$ & & $\checkmark$ & $\checkmark$ & & \\
\hline & Osteobrama cotio & NT & $\mathrm{LC}$ & $\checkmark$ & $\checkmark$ & $\checkmark$ & $\checkmark$ & \\
\hline & Poropuntius clavatus & NE & NT & & $\checkmark$ & & & \\
\hline & Pethia conchonius & $\mathrm{LC}$ & $\mathrm{LC}$ & $\checkmark$ & $\checkmark$ & $\checkmark$ & $\checkmark$ & $\checkmark$ \\
\hline & Pethia phutunio & $\mathrm{LC}$ & $\mathrm{LC}$ & $\checkmark$ & $\checkmark$ & $\checkmark$ & $\checkmark$ & $\checkmark$ \\
\hline & Pethia ticto & VU & $\mathrm{LC}$ & $\checkmark$ & $\checkmark$ & $\checkmark$ & $\checkmark$ & $\checkmark$ \\
\hline & Puntius chola & $\mathrm{LC}$ & $\mathrm{LC}$ & & $\checkmark$ & $\checkmark$ & $\checkmark$ & $\checkmark$ \\
\hline & Puntius gelius & NT & $\mathrm{LC}$ & & $\checkmark$ & & & \\
\hline & Puntius sophore & $\mathrm{LC}$ & $\mathrm{LC}$ & $\checkmark$ & $\checkmark$ & $\checkmark$ & $\checkmark$ & $\checkmark$ \\
\hline & Puntius terio & $\mathrm{LC}$ & $\mathrm{LC}$ & & & $\checkmark$ & & $\checkmark$ \\
\hline & Systomus sarana & NT & $\mathrm{LC}$ & $\checkmark$ & $\checkmark$ & $\checkmark$ & $\checkmark$ & $\checkmark$ \\
\hline & $\begin{array}{l}\text { Psilorhynchus } \\
\text { sucatio }\end{array}$ & NT & $\mathrm{LC}$ & & $\checkmark$ & & & \\
\hline & Raiamas bola & EN & $\mathrm{LC}$ & & $\checkmark$ & $\checkmark$ & & \\
\hline & Rasbora rasbora & NT & $\mathrm{LC}$ & & & $\checkmark$ & & \\
\hline Cyprinidae & Rasbora daniconius & $\mathrm{LC}$ & $\mathrm{LC}$ & & $\checkmark$ & $\checkmark$ & & \\
\hline & $\begin{array}{l}\text { Salmostoma } \\
\text { acinaces }\end{array}$ & $\mathrm{LC}$ & $\mathrm{LC}$ & & $\checkmark$ & & & \\
\hline & Salmostoma bacaila & $\mathrm{LC}$ & $\mathrm{LC}$ & $\checkmark$ & $\checkmark$ & $\checkmark$ & $\checkmark$ & \\
\hline & Salmostoma phulo & NT & $\mathrm{LC}$ & $\checkmark$ & $\checkmark$ & $\checkmark$ & & \\
\hline & Securicula gora & NT & $\mathrm{LC}$ & $\checkmark$ & $\checkmark$ & $\checkmark$ & & \\
\hline Ambassidae & Chanda nama & $\mathrm{LC}$ & $\mathrm{LC}$ & $\checkmark$ & $\checkmark$ & $\checkmark$ & $\checkmark$ & \\
\hline & Parambassis lala & $\mathrm{LC}$ & $\mathrm{NE}$ & $\checkmark$ & & & $\checkmark$ & \\
\hline & Parambassis ranga & $\mathrm{LC}$ & $\mathrm{LC}$ & $\checkmark$ & $\checkmark$ & $\checkmark$ & $\checkmark$ & \\
\hline & $\begin{array}{l}\text { Pseudambassis } \\
\text { baculis }\end{array}$ & NT & $\mathrm{LC}$ & & $\checkmark$ & $\checkmark$ & & \\
\hline Anabantidae & Anabas testudineus & $\mathrm{LC}$ & DD & $\checkmark$ & $\checkmark$ & $\checkmark$ & $\checkmark$ & $\checkmark$ \\
\hline Badidae & Badis badis & NT & $\mathrm{LC}$ & $\checkmark$ & $\checkmark$ & $\checkmark$ & $\checkmark$ & $\checkmark$ \\
\hline Nandidae & Nandus nandus & NT & $\mathrm{LC}$ & $\checkmark$ & $\checkmark$ & $\checkmark$ & $\checkmark$ & $\checkmark$ \\
\hline Osphronemidae & Ctenops nobilis & $\mathrm{LC}$ & NT & & & $\checkmark$ & & \\
\hline & Trichogaster chuna & $\mathrm{LC}$ & $\mathrm{LC}$ & $\checkmark$ & $\checkmark$ & $\checkmark$ & $\checkmark$ & $\checkmark$ \\
\hline & Trichogster fasciata & $\mathrm{LC}$ & $\mathrm{LC}$ & $\checkmark$ & $\checkmark$ & $\checkmark$ & $\checkmark$ & $\checkmark$ \\
\hline & Trichogaster labiosa & $\mathrm{NE}$ & $\mathrm{LC}$ & & & $\checkmark$ & & \\
\hline & Trichogaster lalia & $\mathrm{LC}$ & $\mathrm{LC}$ & $\checkmark$ & $\checkmark$ & $\checkmark$ & $\checkmark$ & $\checkmark$ \\
\hline Mugilidae & Rhinomugil corsula & $\mathrm{LC}$ & $\mathrm{LC}$ & & $\checkmark$ & & & \\
\hline & Sicamugil cascasia & VU & $\mathrm{LC}$ & & $\checkmark$ & & & \\
\hline Gobiidae & Glossogobius giuris & $\mathrm{LC}$ & $\mathrm{LC}$ & $\checkmark$ & $\checkmark$ & $\checkmark$ & $\checkmark$ & $\checkmark$ \\
\hline Mastacembelidae & $\begin{array}{l}\text { Macrognathus } \\
\text { pancalus }\end{array}$ & $\mathrm{LC}$ & $\mathrm{LC}$ & $\checkmark$ & $\checkmark$ & $\checkmark$ & $\checkmark$ & $\checkmark$ \\
\hline Mastacembelidae & $\begin{array}{l}\text { Macrognathus } \\
\text { aculeatus }\end{array}$ & NT & $\mathrm{NE}$ & $\checkmark$ & $\checkmark$ & $\checkmark$ & $\checkmark$ & $\checkmark$ \\
\hline & $\begin{array}{l}\text { Mastacembelus } \\
\text { armatus }\end{array}$ & $\mathrm{EN}$ & $\mathrm{NE}$ & $\checkmark$ & $\checkmark$ & & $\checkmark$ & $\checkmark$ \\
\hline Amblycipitidae & Amblyceps mangois & $\mathrm{LC}$ & $\mathrm{LC}$ & $\checkmark$ & & & & $\checkmark$ \\
\hline Clariidae & Clarias batrachus & $\mathrm{LC}$ & $\mathrm{LC}$ & $\checkmark$ & $\checkmark$ & $\checkmark$ & $\checkmark$ & $\checkmark$ \\
\hline Chacidae & Chaca chaca & $\mathrm{EN}$ & $\mathrm{LC}$ & $\checkmark$ & $\checkmark$ & $\checkmark$ & $\checkmark$ & $\checkmark$ \\
\hline Bagridae & Batasio batasio & NT & $\mathrm{LC}$ & & $\checkmark$ & & & \\
\hline
\end{tabular}




\begin{tabular}{|c|c|c|c|c|c|c|c|c|}
\hline & Batasio tengana & EN & $\mathrm{LC}$ & & $\checkmark$ & & & \\
\hline & Hemibagrus menoda & NT & $\mathrm{LC}$ & $\checkmark$ & $\checkmark$ & $\checkmark$ & $\checkmark$ & \\
\hline & Mystus armatus & DD & $\mathrm{LC}$ & & $\checkmark$ & & & \\
\hline & Mystus bleekeri & $\mathrm{LC}$ & $\mathrm{LC}$ & & $\checkmark$ & $\checkmark$ & & $\checkmark$ \\
\hline & Mystus cavasius & NT & $\mathrm{LC}$ & $\checkmark$ & $\checkmark$ & $\checkmark$ & $\checkmark$ & \\
\hline & Mystus tengara & $\mathrm{LC}$ & $\mathrm{LC}$ & $\checkmark$ & $\checkmark$ & $\checkmark$ & $\checkmark$ & $\checkmark$ \\
\hline & Mystus vittatus & $\mathrm{LC}$ & $\mathrm{LC}$ & $\checkmark$ & $\checkmark$ & $\checkmark$ & & $\checkmark$ \\
\hline & Rama chandramara & $\mathrm{LC}$ & $\mathrm{LC}$ & & $\checkmark$ & & $\checkmark$ & \\
\hline & Rita rita & EN & $\mathrm{LC}$ & $\checkmark$ & $\checkmark$ & & $\checkmark$ & $\checkmark$ \\
\hline & Sperata aor & VU & $\mathrm{LC}$ & $\checkmark$ & $\checkmark$ & & $\checkmark$ & $\checkmark$ \\
\hline & Sperata seenghala & VU & $\mathrm{LC}$ & $\checkmark$ & $\checkmark$ & & $\checkmark$ & $\checkmark$ \\
\hline Erethestidae & Conta conta & NT & $\mathrm{NE}$ & & $\checkmark$ & & & \\
\hline & Hara hara & $\mathrm{LC}$ & $\mathrm{LC}$ & $\checkmark$ & $\checkmark$ & & & $\checkmark$ \\
\hline & Hara jerdoni & $\mathrm{LC}$ & $\mathrm{LC}$ & & $\checkmark$ & & & \\
\hline & $\begin{array}{l}\text { Erethistoides } \\
\text { infuscatus }\end{array}$ & $\mathrm{NE}$ & $\mathrm{DD}$ & & $\checkmark$ & & & \\
\hline Erethestidae & Pseudolaguvia shawi & DD & DD & & $\checkmark$ & & & \\
\hline & $\begin{array}{l}\text { Pseudolaguvia } \\
\text { inornata }\end{array}$ & DD & DD & & $\checkmark$ & & & \\
\hline Erethestidae & $\begin{array}{l}\text { Pseudolaguvia } \\
\text { murikata }\end{array}$ & DD & DD & & $\checkmark$ & & & \\
\hline $\begin{array}{l}\text { Heteropneusteida } \\
\mathrm{e}\end{array}$ & $\begin{array}{l}\text { Heteropneustes } \\
\text { fossilis }\end{array}$ & $\mathrm{LC}$ & $\mathrm{LC}$ & $\checkmark$ & $\checkmark$ & $\checkmark$ & $\checkmark$ & $\checkmark$ \\
\hline Pangasiidae & Pangasius pangasius & EN & $\mathrm{LC}$ & & $\checkmark$ & & $\checkmark$ & \\
\hline Schilbeidae & Ailia coila & $\mathrm{LC}$ & NT & $\checkmark$ & $\checkmark$ & $\checkmark$ & $\checkmark$ & $\checkmark$ \\
\hline & Ailia punctata & $\mathrm{LC}$ & $\mathrm{NE}$ & & $\checkmark$ & $\checkmark$ & $\checkmark$ & \\
\hline & Clupisoma garua & $\mathrm{EN}$ & $\mathrm{NE}$ & $\checkmark$ & $\checkmark$ & & $\checkmark$ & $\checkmark$ \\
\hline & Eutropiichthys vacha & $\mathrm{LC}$ & $\mathrm{LC}$ & $\checkmark$ & $\checkmark$ & $\checkmark$ & $\checkmark$ & $\checkmark$ \\
\hline & $\begin{array}{l}\text { Pseudotropius } \\
\text { atherinoides }\end{array}$ & $\mathrm{LC}$ & $\mathrm{LC}$ & $\checkmark$ & $\checkmark$ & $\checkmark$ & $\checkmark$ & $\checkmark$ \\
\hline Siluridae & Ompok bimaculatus & EN & NT & $\checkmark$ & $\checkmark$ & $\checkmark$ & & $\checkmark$ \\
\hline & Ompok pabda & $\mathrm{EN}$ & NT & $\checkmark$ & $\checkmark$ & $\checkmark$ & $\checkmark$ & $\checkmark$ \\
\hline & Ompok pabo & CR & NT & $\checkmark$ & $\checkmark$ & & $\checkmark$ & \\
\hline & Silonia silondia & $\mathrm{LC}$ & $\mathrm{LC}$ & & $\checkmark$ & $\checkmark$ & & \\
\hline & Wallago attu & VU & NT & $\checkmark$ & $\checkmark$ & & $\checkmark$ & $\checkmark$ \\
\hline & Bagarius bagarius & $\mathrm{CR}$ & NT & $\checkmark$ & $\checkmark$ & & & $\checkmark$ \\
\hline & Gagata cenia & $\mathrm{LC}$ & $\mathrm{LC}$ & & $\checkmark$ & $\checkmark$ & $\checkmark$ & \\
\hline Sisoridae & Gagata gagata & $\mathrm{LC}$ & $\mathrm{LC}$ & & $\checkmark$ & & & \\
\hline & Gagata youssoufi & NT & $\mathrm{LC}$ & & $\checkmark$ & $\checkmark$ & & \\
\hline & $\begin{array}{l}\text { Gogangra } \\
\text { viridescens }\end{array}$ & $\mathrm{LC}$ & $\mathrm{LC}$ & & $\checkmark$ & & & \\
\hline & $\begin{array}{l}\text { Glyptothorax } \\
\text { telchitta }\end{array}$ & VU & $\mathrm{LC}$ & $\checkmark$ & & $\checkmark$ & $\checkmark$ & \\
\hline Sisoridae & $\begin{array}{l}\text { Parachiloglanis } \\
\text { hodgarti }\end{array}$ & NE & $\mathrm{LC}$ & & $\checkmark$ & & & \\
\hline Belonidae & Xenentodon cancila & LC & NE & $\checkmark$ & $\checkmark$ & $\checkmark$ & $\checkmark$ & $\checkmark$ \\
\hline Hemiramphidae & $\begin{array}{l}\text { Hyporhamphus } \\
\text { limbatus }\end{array}$ & $\mathrm{LC}$ & $\mathrm{NE}$ & & $\checkmark$ & & & \\
\hline Tetraodontidae & Chelonodon patoca & DD & NE & & $\checkmark$ & $\checkmark$ & & \\
\hline & Tetraodon cutcutia & $\mathrm{LC}$ & $\mathrm{LC}$ & $\checkmark$ & $\checkmark$ & $\checkmark$ & $\checkmark$ & \\
\hline Channidae & Channa marulius & EN & $\mathrm{LC}$ & $\checkmark$ & $\checkmark$ & $\checkmark$ & $\checkmark$ & $\checkmark$ \\
\hline & Channa orientalis & $\mathrm{LC}$ & $\mathrm{LC}$ & $\checkmark$ & $\checkmark$ & $\checkmark$ & $\checkmark$ & $\checkmark$ \\
\hline & Channa punctata & $\mathrm{LC}$ & $\mathrm{LC}$ & $\checkmark$ & $\checkmark$ & $\checkmark$ & $\checkmark$ & $\checkmark$ \\
\hline
\end{tabular}

ECOPRINT VOL 24，2017 


\begin{tabular}{|c|c|c|c|c|c|c|c|}
\hline \multirow{4}{*}{$\begin{array}{l}\text { Exotic specie } \\
\text { Cyprinidae }\end{array}$} & \multirow{2}{*}{$\begin{array}{l}\text { Channa gachua } \\
\text { Channa striata }\end{array}$} & \multirow{2}{*}{$\begin{array}{l}\mathrm{LC} \\
\mathrm{LC}\end{array}$} & \multirow{2}{*}{$\begin{array}{l}\mathrm{LC} \\
\mathrm{LC}\end{array}$} & \multicolumn{4}{|c|}{$\checkmark$} \\
\hline & & & & $\checkmark$ & $\checkmark$ & $\checkmark$ & $\checkmark$ \\
\hline & & & & & & & \\
\hline & Aristichthys nobilis & $\mathrm{LC}$ & $\mathrm{LC}$ & $\checkmark$ & & $\checkmark$ & $\checkmark$ \\
\hline \multirow{4}{*}{ Cyprinidae } & $\begin{array}{l}\text { Ctenopharyngodon } \\
\text { idella }\end{array}$ & $\mathrm{LC}$ & $\mathrm{LC}$ & $\checkmark$ & & $\checkmark$ & $\checkmark$ \\
\hline & Cyprinus carpio & $\mathrm{LC}$ & $\mathrm{LC}$ & $\checkmark$ & & $\checkmark$ & $\checkmark$ \\
\hline & $\begin{array}{l}\text { Hypopthalmichthys } \\
\text { molitrix }\end{array}$ & $\mathrm{LC}$ & $\mathrm{LC}$ & $\checkmark$ & & $\checkmark$ & $\checkmark$ \\
\hline & $\begin{array}{l}\text { Barbonymus } \\
\text { goninotus }\end{array}$ & $\mathrm{LC}$ & $\mathrm{LC}$ & $\checkmark$ & & $\checkmark$ & $\checkmark$ \\
\hline \multirow[t]{2}{*}{ Cichlidae } & $\begin{array}{l}\text { Oreochromis } \\
\text { mossambicus }\end{array}$ & $\mathrm{LC}$ & $\mathrm{LC}$ & $\checkmark$ & & $\checkmark$ & $\checkmark$ \\
\hline & $\begin{array}{l}\text { Oreochromis } \\
\text { niloticus }\end{array}$ & $\mathrm{LC}$ & $\mathrm{LC}$ & $\checkmark$ & & $\checkmark$ & $\checkmark$ \\
\hline Pangasiidae & $\begin{array}{l}\text { Pangasius } \\
\text { hypophthalmus }\end{array}$ & $\mathrm{LC}$ & $\mathrm{LC}$ & $\checkmark$ & & $\checkmark$ & $\checkmark$ \\
\hline
\end{tabular}

We found 9 common exotic species reported Galib et al. (2009), Siddiqui et al. (2016) and Rahman et al. (2017). However, we observed Thick-lipped Gourami Trichogaster labiosa was enlisted by Kostori et al. (2011). This fish species is originated in Southeast Asia, mentioned nowhere in the wilderness of Bangladesh and seems to be an additional for the list of exotic fish species of the country. However, this requires further sampling effort and investigation.

Hossain et al. (2009) reported three species from the Chalan Beel that have been observed in no national fish inventories of Bangladesh - not in Siddiqui et al. (2008) and as recent as in IUCN Bangladesh (2015). They are appeared to be new inclusion for the entire country; viz., Stedman barb Poropuntius clavatus, Torrent catfish Parachiloglanis hodgarti and an erethistid catfish Erethistoides infuscatus. Reports of these species call on an immediate rigorous sampling effort in the Chalan Beel area. According to $\mathrm{Ng}$ (2006), Devi and Boguskaya (2009), Dahanukar (2010), range of all three species encompasses the North Bengal and overlaps Bangladesh.

In this review, categorization of fish species, analysis of their frequency of occurrence and comparison among the different inventories and using meta-analysis to describe the drift shed lights on the present status of the Chalan Beel fish inventories. We can see that the Chalan Beel is home to half of the documented freshwater fish species of Bangladesh; many of which, apart from being exquisite diminutive seldom reported rarities, are threatened with extinction. Despite the surviving richness, since 1982, and the fish population of the Chalan Beel has been halved due to anthropogenic causes such as built up areas, agrochemicals and road encroachments (AFP 2010). We recommend that systematic ecological surveys and subsequent declaration of sanctuaries should be targeted to ensure secured future and sustainability of country's key ichthyofaunal hotpsot.

\section{REFERENCES}

AFP. 2010. Bangladesh's once plentiful rivers run low on fish. Agence France-Presse, The Independent. London. Accessed on: 21 April 2018.

https://web.archive.org/web/20150722075535/ http:/www.independent.co.uk/life-style/healthand-families/bangladeshs-once-plentifulrivers-run-low-on-fish-2169547.html

Alam, M.S. and Hossain, M.S. 2003. Chalan Beel. in S. Islam, S. Miah, W. Ahmed, A.M. Chawdhury, S.M.M. Rahman, K. Siddiqui, S.M.H. Kabir, A. Kabir, K.M.H. Huq, F.

ECOPRINT VOL 24, 2017 
Alam, B.A. Kamal, S. Akhter (eds.) Banglapedia. Asiatic Society of Bangladesh. http://en.banglapedia.org/index.php?title=Chal an_Beel

Dahanukar, N. 2010. Poropuntius clavatus. The IUCN Red List of Threatened Species 2010: e.T166589A6242148. http://dx.doi.org/10.2305/IUCN.UK.20104.RLTS.T166589A6242148.en. Downloaded on 27 April 2018.

Devi, R. and Boguskaya, N. 2009. Parachiloglanis hodgarti. The IUCN Red List of Threatened Species 2009: e.T166618A6248603. http://dx.doi.org/10.2305/IUCN.UK.20092.RLTS.T166618A6248603.en. Downloaded on 27 April 2018.

DoF. 2016. Fisheries Statistical Report of Bangladesh 2014-2015. Fisheries Resources Survey System, Department of Fisheries, the Ministry of Fisheries and Livestock, the Government of People's Republic of Bangladesh, Dhaka, Bangladesh. 57 pp.

Eschmeyer, W.N. and Fong, J.D. 2018. Catalog of fishes. Species by family/subfamily. http://researcharchive.calacademy.org/research /ichthyology/catalog/SpeciesByFamily.asp\#Ba didae. Accessed on: 2018-4-21.

Froese, R. and Pauly. D. (eds.) 2018. FishBase. World Wide Web electronic publication. www.fishbase.org, version (02/2018). Accessed on: 2018-4-21.

Galib, S.M., Samad, M.A., Mohsin, A.B.M., Flowra, F.A. and Alam, M.T. 2009. Present status of fishes in the Chalan Beel - The largest beel (wetland) of Bangladesh. Int. J. Anim. Fish. Sci. 2(3): 214-218.

Hossain, M.A.R., Nahiduzzaman, M., Sayeed, M.A., Azim, M.E., Wahab, M.A. and Lin, P.G. 2009. The Chalan Beel in Bangladesh: habitat and biodiversity degradation and implication for future management. Lake. Reserv. Manage. 14:3-19.

IUCN Bangladesh. 2015. Red List of Bangladesh Vol. 5: Freshwater Fishes. IUCN, International Union for Conservation of Nature, Bangladesh Country Office, Dhaka, Bangladesh, pp. xvi+360.

Kostori, F.A., Parween, S. and Islam, M.N. 2011. Availability of small indigenous species (SIS) of fish in the Chalan Beel - the largest wetland of Bangladesh. Univ. J. Zool. Rajshahi Univ. 30: $67-72$.

Kullander, S.O., Rahman, M.M., Noren, M. and Mollah, A.R. 2015. Danio annulosus, a new species of chain Danio from the Shuvolong Falls in Bangladesh (Teleostei: Cyprinidae: Danioninae). Zootaxa 3994(1): 053-068.

Nelson, J.S., Grande, T.C. and Wilson, M.V. 2016. Fishes of the World. John Wiley \& Sons, New Jersey, 395pp.

Ng, H.H. 2005. Two new species of Pseudolaguvia (Teleostei: Erethistidae) from Bangladesh. Zootaxa 1044(1): 35-47.

Ng, H.H. 2006. Erethistoides infuscatus, a new species of catfish (Teleostei: Erethistidae) from South Asia. Ichthyol. Explor. Freshw. 17(3): 281-287.

Rahman, M.M., Mollah, A.R., Norén, M. and Kullander, S.O. 2016. Garra mini, a new small species of rheophilic cyprinid fish (Teleostei: Cyprinidae) from southeastern hilly areas of Bangladesh. Ichthyol. Explor. Freshw. 27(2): 173-181.

Rahman M.M., M.M. Hoque, R. Karim and M.M. Sarker 2017. Present status and scope of fisheries culture at Chalan Beel in Bangladesh. Int. J. Adv. Res. Biol. Sci. 4 (6): 34-40. 
Rahman, M.A., Akter, S., Haider, M.I., Majumder, M.W.R. 2017. Present Status (Biodiversity and Conservation) of fish at Chalan Beel. Bangladesh. Int. J. Zool.Stud. 2(2): 31-37.

Sultana, N. and Islam M.N. 2016. Fish Production and Livelihood of Fish Farmers of Chalan Beel Area, Bangladesh. J. Agric. Vet. Sci. 9: 51-56.

Siddique, M.A.B., Hussain, M.A., Flowra, F.A. and Alam, M.M. 2016. Assessment of fish fauna in relation to biodiversity indices of
Chalan Beel, Bangladesh. Int. J. Aquat. Biol. 4(5): 345-352.

Siddiqui, K.U., Islam, M.A., Kabir, S.M.H., Ahmad, M., Ahmed, A.T.A., Rahman, A.K.A., Haque, E.U., Ahmed, Z.U., Begum, Z.N.T., Hasan, M.A., Khondker. M. and Rahman, M.M. (eds.). 2008. Encyclopedia of Flora and Fauna of Bangladesh. Vol. 23. Freshwater Fishes. Asiatic Society of Bangladesh, Dhaka, 334. 